\title{
Bioavailability and antioxidant effect of ergothioneine in human blood
}

\author{
Leandra Kiani Schmitz \\ Maastricht University \\ Ikemm.schmitz@student.maastrichtuniversity.nl
}

\section{Abstract}

Edible mushrooms, such as the white button mushrooms contain several biological compounds including ergothioneine (ET). The main function of ET seems to be to protect cells from oxidative stress. Although humans cannot synthesize ET, the human body does have its own ergothioneine transporter (ETT), which allows cells, for instance red blood cells (RBCs), to take up and store ET. The existence of this transporter suggests that ET might have a physiological importance in humans. The aims of this study were to examine the availability of ET in RBCs and the protective effect of ET on oxidative stress in RBCs. The uptake of ET in RBCs was studied in vitro by treating RBCs with known concentrations of ET $(0-100 \mu M)$. The amount of ET in the RBCs was determined using high performance liquid chromatography (HPLC). The protective effect of ET was studied by measuring the haemolysis in oxidative stress induced RBCs, which were pre-treated with ET in concentrations ranging from $0-100 \mu \mathrm{M}$. In addition, a pilot study was performed where $10,6 \mathrm{mg}$ ET was administered to subjects $(n=6)$ through a mushroom soup. Before and three hours after consumption, blood samples were collected. The blood samples were analyzed to determine the concentration ET in the RBCs using HPLC and the protective effect of ET was studied the same way as for in vitro. Main findings were that ET was taken up in RBCs after treatment with all ET concentrations, but only after 30 minutes and two hours. Furthermore, RBCs pre-treated with ET had less haemolysis than nontreated RBCs; pre-treatment with $100 \mu \mathrm{M}$ ET was observed to significantly $(p<0,016)$ decrease haemolysis in comparison with the non-treated RBCs. Consumption of the mushroom soup did not result in increased ET levels in the RBCs, although three out of five subjects had less haemolysis compared to consumption of the placebo soup. However, the beneficial effects after consumption of the mushroom soup cannot be assigned to ET for sure; other unobserved compounds could be involved. 
Keywords

Ergothioneine, mushrooms, bioavailability, haemolysis, antioxidant.

\section{Introduction}

Edible mushrooms, such as the white button mushroom, have a high nutritional value. They contain several biological compounds, including ergothioneine (ET), a stable intracellular antioxidant (1-3). The most significant function of ET seems to be to protect cells from oxidative stress $(2,4)$. Humans cannot synthesize ET and thus can only obtain it from food $(2,3)$. However, we do have an unique ergothioneine transporter (ETT), which facilitates the specific uptake of ET $(5,6)$. ET is taken up by RBCs, but also kidney, cerebellum, monocytes and lung cells. The existence of this specific transporter suggests a physiological importance in human health.

This study evaluates the bioavailability of ET in human RBCs and the possible protective effect of ET on oxidative stress in human RBCs. Assays are performed in in vitro and in vivo samples. In vitro assays will be studied in human RBCs treated with known concentrations ET (o-100 $\mu \mathrm{M})$. In vivo samples are conducted from a pilot study, in which subjects eat a bouillon soup, once with and once without mushrooms. These pilot study samples will be partially used in an ex-vivo assay.

It is hypothesized that ET will be taken up in RBCs in the in vitro and in vivo studies and that the ET concentration in the RBCs will increase after treatment with different concentration ET and after consumption of the mushroom soup. It is also expected that ET will decrease the oxidative damage in RBCs.

\section{Material and methods}

Whole blood was collected from human donors by venapuncture in heparinised tubes and centrifuged to obtain the RBCs. RBCs were washed with phosphate buffered saline (PBS) and used for various assays.

Treatment of ergothioneine

For the in vitro studies, human RBCs were dissolved in RPMI medium to get $20 \%$ hematocrit solution, then the RBCs in solution were treated with different concentrations $\mathrm{ET}(0,10,50$, $100 \mu \mathrm{M}$ ) for 4 hours at $37^{\circ} \mathrm{C}$. The treated cells were used for various assays. 


\section{Quantification of ergothioneine by HPLC}

Quantification of ET was carried out using an Agilent/HP 1100 series HPLC system. After treatment with $\mathrm{ET}$, samples of the different concentrations were taken after 30 minutes, 2 and 4 hours. Samples were centrifuged and the RPMI medium was removed. RBCs were once washed and then extraction solution (10 nm DTT in 0,1 $\mathrm{mM}$ phosphate buffer, $\mathrm{pH}$ 8) was added 1:1 to the washed treated RBCs. Samples were lysed in $90^{\circ} \mathrm{C}$, centrifuged at maximal speed and the supernatant was collected for HPLC analysis. An Alltima ${ }^{\mathrm{TM}} \mathrm{C}_{18}$ 5 column ( $250 \mathrm{~mm} \times 3.0 \mathrm{~mm}$; serial number 209070078) was used to separate ET and detected with UV-Vis (Agilent 1100), with an isocratic mobile phase of $0,3 \mathrm{M}$ phosphate buffer, $\mathrm{pH} 7,3$ and $1 \%$ acetonitrile. Separation was carried out with a flow rate of $0,5 \mathrm{ml} / \mathrm{min}$ and injection volume was set at $10 \mu \mathrm{l}$ with an ambient column temperature. Absorbance was measured at $254 \mathrm{~nm}$ using a HP1100 series variable wavelength detector (VWD).

\section{Protective effect of ergothioneine against t-BHP induced haemolysis}

After pre-treatment for four hours with ET, a sample was taken of the treated RBCs and centrifuged. RPMI medium was removed and the RBCs were once washed with PBS. RBCS were diluted to a $5 \% \mathrm{v} / \mathrm{v}$ suspension with PBS. Samples were then incubated with PBS (control), 0,1\% Triton $\mathrm{X}-100$ (maximum haemolysis) or $3 \mathrm{mM}$ tert-butyl hydroperoxide (t-BHP) for two hours at $37^{\circ} \mathrm{C}$. After two hours, samples were removed and centrifuged. The absorbance of the supernatant was measured spectrophotometrically at $540 \mathrm{~nm}$. The average absorbance was used and the percentage haemolysis was calculated as follow:

$$
\frac{\text { absorbance of sample } t-B H P-\text { absorbance control }}{\text { absorbance of sample Triton } X-100} \times 100
$$

Pilot study

This pilot study employed a randomized, 2-period, crossover study design. Healthy subjects $(n=6)$ between the age of 20-40 years with a body mass index (BMI) between 20 and $30 \mathrm{~kg} / \mathrm{m}^{2}$ were studied. The study involved two test days in which subjects consumed a bouillon soup, once with and once without $\sim 355$ grams of white button mushrooms. Prior each test day, subjects were not allowed to perform extensive sports and after 10 p.m., they were only allowed to drink water or tea. On the test days, subjects were overnight fasted and at the start of the test day, one tube of blood (baseline) was collected. Subjects then eat the appointed soup and three hours after consumption, a second tube of blood was withdrawn. Blood was drawn by venapuncture and collected in sodium heparin tubes. 
The collected blood was prepared for HPLC to analyze the concentration of ET in the RBCs, as described above. Next to that, RBCs were used in an ex vivo study in which the t-BHP induced haemolysis assay was applied.

\section{Results}

\section{Quantification of ergothioneine}

After treatment with o, 10, 50 and $100 \mu \mathrm{M} \mathrm{ET}$, samples were taken after 30 minutes, 2 and 4 hours. It was observed that the non-treated samples, the RBCs already contained ET (figure 1a). It was also observed that the highest concentrations ET in the RBCs were after 30 minutes of treatment with 10,50 and $100 \mu \mathrm{M}$ ET (figure $1 \mathrm{~b}-\mathrm{d}$ ). A decreasing tendency was observed after two and four hours with the lowest ET concentration in the RBCs after four hours for all treatments. The treatment of the RBCs with concentrations ET ranging from o-100 $\mu \mathrm{M}$ for up to four hours did not lead to statistically significant differences in intracellular concentration of ET (figure 1a-d).

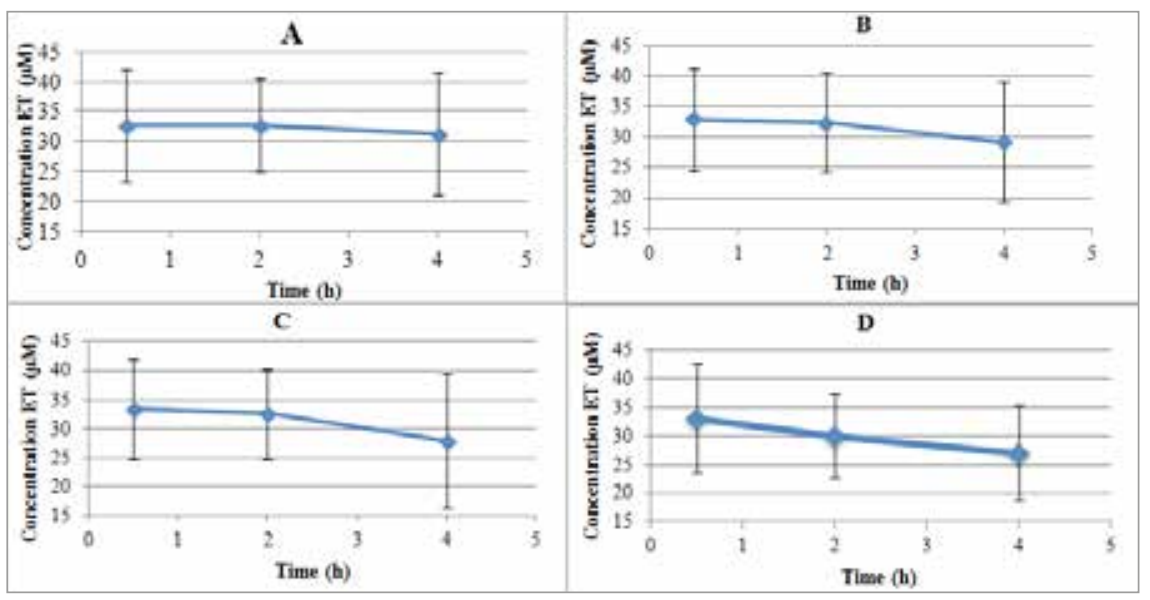

Figure 1.Time course of the ET concentration $(\mu M)$ in RBCs incubated with (A) $\circ \mu M$, (B) $10 \mu M$, (C) $50 \mu M$ and (D) $100 \mu \mathrm{M}$. Results are shown as average \pm SD of duplicate measurements from three different donors

An increasing tendency in concentration ET in the RBCs was observed after 30 minutes and 2 hours with treatment of 10 and $50 \mu \mathrm{M} \mathrm{ET} \mathrm{compared} \mathrm{to} \mathrm{the} \mathrm{non-treated} \mathrm{cells.} \mathrm{Treatment}$ with $100 \mu \mathrm{M}$ ET tended to decrease after 30 minutes and 2 hours compared to $50 \mu \mathrm{M}$ ET (figure 2a-b). After 4 hours of treatment, concentrations ET in RBCs treated with 10, 50 and $100 \mu \mathrm{M}$ ET tended to be decreased compared to non-treated RBCs (figure $2 \mathrm{c}$ ). 


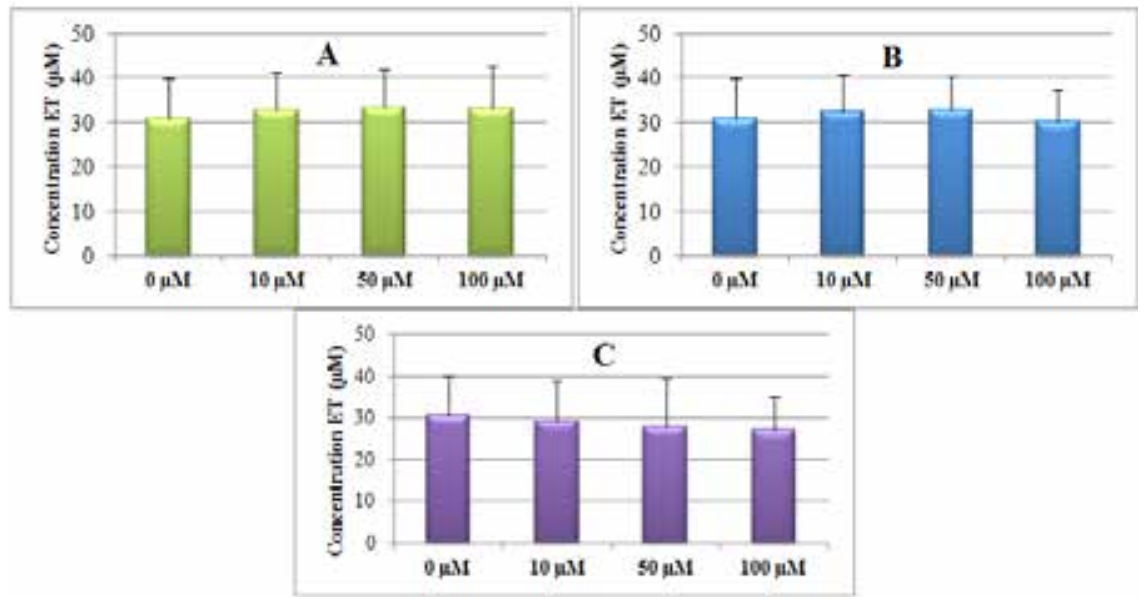

Figure 2. ET concentration ( $\mu M$ ) in RBCs treated with $E T$ at concentrations ranging from $o-100 \mu M$ for $(A)$ 30 minutes, (B) 2 hours, (C) 4 hours. Results are presented as the average \pm SD of duplicate measurements from three different donors

\section{Protective effect of ergothioneine against t-BHP induced haemolysis}

To measure the effect of ET on oxidative damage of RBCs, pre-treated RBCs with $0,10,50$ and $100 \mu \mathrm{M} \mathrm{ET}$, were incubated with $3 \mathrm{mM} \mathrm{t}-\mathrm{BHP}$ for two hours to induce oxidative stress and haemolysis was measured. The average haemolysis of the three donors tended to be decreased in the ET treated cells compared with the non-treated cells. It was observed that the higher the treatment of RBCs with ET was, the lower the haemolysis of the RBCs was, however this regression was not statistically significant $(p=0,102)$. After treatment with $100 \mu \mathrm{M}$, haemolysis did significantly decrease compared to the non-treated cells $(p=0,016)$ (figure 3).

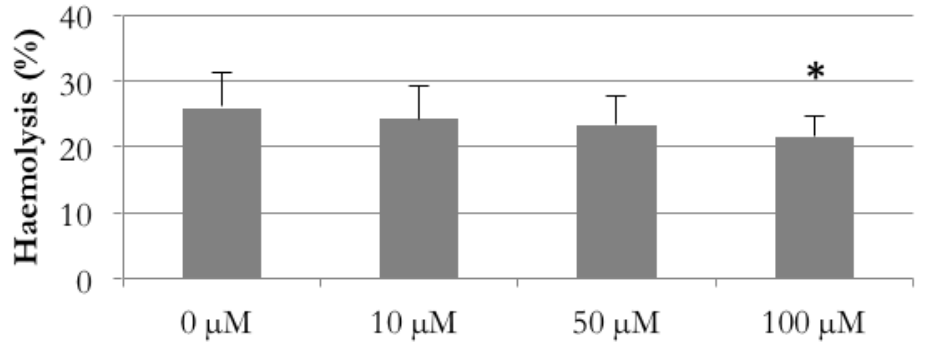

Figure 3. Haemolysis of RBCs after treatment with ET at concentrations ranging from $0-100 \mu M$ for four hours. Haemolysis was calculated as \% of maximal haemolysis induced by treatment of the RBCs with Triton $\mathrm{X}-100$. Results are presented as the average \pm SD of duplicate measurements from three different donors. * means a $p$-value $<0,05$ 


\section{Pilot study}

A before and after sample was collected for every subject on both test days. On the second test day, from one subject no blood could be collected after consumption of the placebo soup and another subject was not able to finish the mushroom soup.

\section{Baseline characteristics}

Study participants $(n=6)$ ranged between 20 and 27 years and there was an equal number of man and women. The mean BMI was $26,8 \mathrm{~kg} / \mathrm{m}^{2}$.

\section{Analysis of mushroom soup}

To analyze the amount of ET in the mushroom soup, the mushroom soup was freeze-dried and prepared to analyze with the HPLC. The measured concentration of the samples was $56,4 \mu \mathrm{M}$ ET. The exact amount of ET in the soup could be calculated and was 10,6 mg per $500 \mathrm{~g}$ of mushroom soup.

\section{Ergothioneine concentration in RBCs}

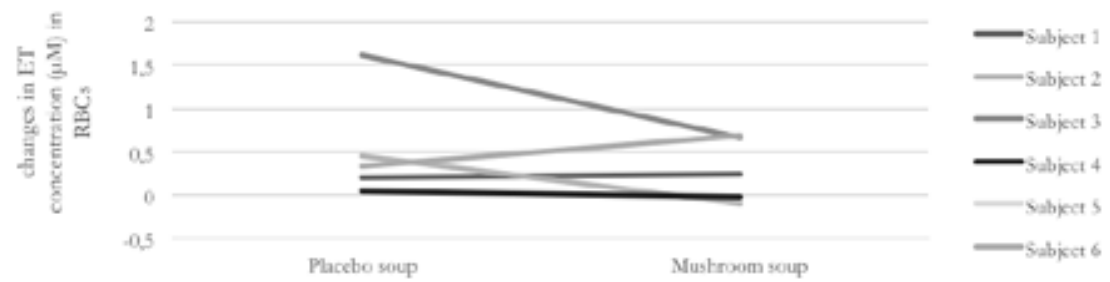

Figure 4. The changes in ET concentration $(\mu M)$ in RBCs after consumption of the placebo soup and after consumption of the mushroom soup. The change was calculated as the concentration of ET in the RBCs after consumption minus the concentration before consumption.

The before and after blood samples of both test days were prepared for HPLC. Concentrations ET in the RBCs were expressed per mmol/L Hb. After consumption of the mushroom soup, concentrations of ET were observed to vary considerably between the different subjects, with two subjects showing an increase, two showing a decrease and one showing no change compared to the placebo soup. No significant differences were found between consumption of the soups and ET concentration in RBCs (figure 4).

\section{Protective effect of ergothioneine}

The before and after blood samples of both test days were treated with $3 \mathrm{mM} \mathrm{t-BHP}$ and haemolysis was measured. Then, the change between before and after consumption of 
both soups in haemolysis was calculated per person. After consumption of the mushroom soup, it was observed that three out of five subjects had less haemolysis and two subjects had increased levels of haemolysis compared with consumption of the placebo soup. No significant differences were found between consumption of the soups and haemolysis of the RBCs (figure 5).

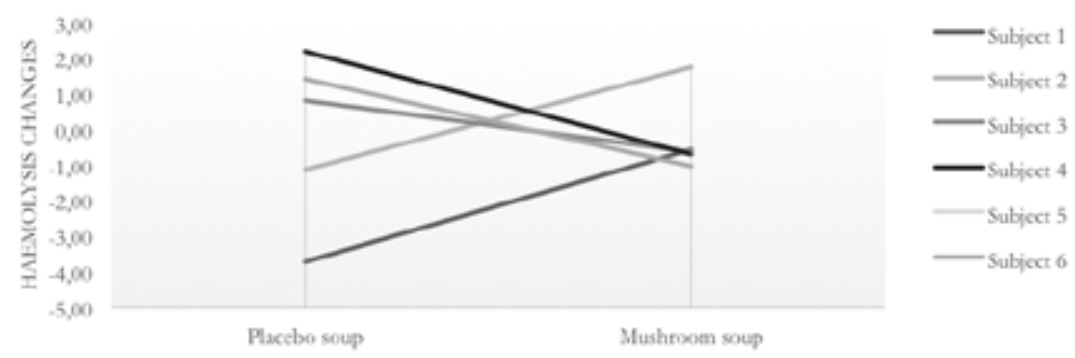

Figure 5. The changes in haemolysis of RBCs after consumption of the placebo soup and after consumption of the mushroom soup. The change was calculated as the haemolysis of RBCs after consumption minus the haemolysis of RBCs before consumption. Haemolysis was calculated as \% of maximal haemolysis, induced by treatment of the RBCs with Triton X-10o. Results are presented as the average of duplicate measurements per subject. There were no results for the fifth subject

\section{Discussion/Conclusion}

The aims of this study were manifold. Firstly, this study examined the availability of ET in RBCs. Secondly, the protective effect of ET on t-BHP-induced haemolysis was examined.

The hypotheses of this study were that ET will be taken up in RBCs in the in vitro and in vivo studies and that the concentration will increase after treatment with different concentrations ET and after consumption of the mushroom soup. In addition, it was expected that ET would decrease the oxidative damage in RBCs. Only parts of these hypotheses were proved during this study. After analyzing blood samples, ET was measured, which indicates that it was available in RBCs. However, concentration did only increase after treatment with 10, 50 and $100 \mu \mathrm{M} \mathrm{ET}$, but not after consumption of the mushroom soup. In addition, the protective effect of ET was proven in the in vitro study, in the pilot study the protective effect could also be due to other biological compounds from the mushrooms. 


\section{Quantification of ergothioneine}

The average concentration ET in the RBCs of the three donors showed an expected trend after 30 minutes and two hours (figure 2a-b). Namely, an increasing concentration ET in the erythrocytes when adding more ET. However, after four hours, the concentration in the RBCs decreases after addition of higher ET concentrations (figure $2 \mathrm{C}$ ). A possible explanation could be that the RBCs experienced oxygen damage, thereby releasing ET into the medium.

\section{Protective effect of ergothioneine}

The effect of pre-treatment with increasing concentrations of ET on oxidative stress induced haemolysis in RBCs did show a trend, although not statistically significant. The higher the added concentration of ET, the less haemolysis occurred. After treatment with $100 \mu \mathrm{M} \mathrm{ET}$, haemolysis was significantly lower than the non-treated cells (figure 3). Although, a dose of $100 \mu \mathrm{M}$ ET does cause significantly lower haemolysis in the RBCs, this antioxidant function found in RBCs do not necessarily reflect the same response for the whole human body.

\section{Pilot study}

\section{Ergothioneine concentration in RBCs}

Only for one out of five subjects a clear increase in ET concentration per mmol/L Hb after consumption of the mushroom soup was observed (figure 4). Although this was not expected on forehand, after analyzing the ET concentration in the mushroom soup it could be due to the low amount of ET in the soup.

\section{Protective effect of ergothioneine}

After consumption of the mushroom soup, three out of five subjects had decreased haemolysis and two subjects had increase haemolysis compared to consumption of the placebo soup (figure 5). Although it was observed that three out of five subjects had decreased haemolysis of RBCs, suggesting a positive effect of ET, the effects could not be assigned to ET for sure. Mushrooms still contain other biological compounds such as vitamin C, E and selenium, other antioxidants, which could cause a better protected RBC (2).

To conclude, in this study it was suggested that ET was taken up by RBCs after treatment with known concentrations $\mathrm{ET}(10,50,100 \mu \mathrm{M})$, and that the highest concentration was reached after 30 minutes. In addition, when more ET was added to the RBCs, less haemolysis was observed. This suggests that RBCs are better protected against oxidative 
stress. However, it must be kept in mind that the antioxidant activity found in the RBCs does not necessarily mean that this would also be the same in the whole body.

Consumption of the mushroom soup did not lead to increased concentrations of ET in the RBCs. In addition, three out of five subjects had less haemolysis after consumption of the mushroom soup in comparison with the placebo soup, however it is not excluded that other biological compounds found in the mushrooms cause this effect. Obviously, ET provides many opportunities for research, which is also necessary, especially to examine the effect of ET in vivo. If research can support the beneficial function of ET in human health, it might be able to get a future as a nutraceutical or functional food.

\section{Role of the student}

Leandra Schmitz was an undergraduate student working under the supervision of Dr. G.J. Hageman when the research in this report was performed. The topic was proposed by the supervisor. The assays were performed individually by the student, as well as processing of the results and formulation of the conclusions and the writing.

\section{Acknowledgments}

I wish to thank my supervisor for her suggestions and comments, which helped me in writing my thesis and conducting the research. In addition, I wish to thank the volunteers for their time and effort to complete the pilot study. Without their help, I would not have been able to conduct this research.

\section{References}

1. Valverde ME, Hernandez-Perez T, Paredes-Lopez O. Edible mushrooms: improving human health and promoting quality life. Int J Microbiol. 2015:376387.

2. Feeney MJ, Dwyer J, Hasler-Lewis CM, Milner JA, Noakes M, Rowe S, et al. Mushrooms and Health Summit proceedings. J Nutr. 2014 Jul;144(7):1128S-36S.

3. Woldegiorgis AZ, Abate D, Haki GD, Ziegler GR. Antioxidant property of edible mushrooms collected from Ethiopia. Food Chem. 2014 Aug 15;157:30-6.

4. Weigand-Heller AJ, Kris-Etherton PM, Beelman RB. The bioavailability of ergothioneine from mushrooms (Agaricus bisporus) and the acute effects on antioxidant capacity and biomarkers of inflammation. Prev Med. 2012 May;54 Suppl:S75-8.

5. Pfeiffer C, Bach M, Bauer T, Campos da Ponte J, Schomig E, Grundemann D. Knockout of the ergothioneine transporter ETT in zebrafish results in increased 8-oxoguanine levels. Free Radic Biol Med. 2015 Mar $5 ; 83: 178-85$. 
6. Cheah IK, Ong RL, Gruber J, Yew TS, Ng LF, Chen CB, et al. Knockout of a putative ergothioneine transporter in Caenorhabditis elegans decreases lifespan and increases susceptibility to oxidative damage. Free Radic Res. 2013 Dec;47(12):1036-45. 